\title{
Plate motions, mantle convection and chaos
}

\author{
S. Quéré ${ }^{1} \&$ A. A. Berezin ${ }^{2}$ \\ ${ }^{1}$ University of Toronto, Toronto, Canada \\ ${ }^{2}$ McMaster University, Hamilton, Canada
}

\begin{abstract}
Plate tectonics is the theory that the earth's lithosphere divided into several plates is linked to processes taking place deep inside the Earth. The motion of the plates can be divided into two terms: the horizontal divergence (ridge-trench system) and the radial vorticity (transform faults and plate spin). At present-day, the degree variance of the horizontal divergence presents two peaks at degrees 4 and 5. So far, these two peaks have been explained by (1) the dominant large scale flow that drives the plates $(4000 \mathrm{~km})$ and/or (2) the Pacific plate. In this paper, we propose a new explanation. We compare the two published plate reconstructions that span the Mesozoic and Cenozoic periods by imposing each of them on top of a convective code. While the constructal theory provides a conceptual framework for our modeling, we venture to mention the possibility of a presence of chaotic behavior in the self-organization of plates linked to mantle convection.

Keywords: plate tectonics, mantle convection, plate motion reconstruction, constructal theory, chaos theory.
\end{abstract}

\section{Introduction}

According to the theory of plate tectonics, the lithosphere (the top rigid part of the Earth) is divided into several plates that rotate relatively to each other at the surface of the Earth (fig. 1). Each plate motion is entirely described by its angular motion. As the divergence of the velocity is null, the velocity field can be described in an unique way by two complementary and orthogonal fields, namely the poloidal $(\phi)$ and toroidal $(\psi)$ fields.

$$
\vec{v}=-1 / \rho_{s}(\underbrace{\overrightarrow{\operatorname{rot}} \overrightarrow{\text { rot }}\left(\phi \frac{\vec{g}}{g}\right)}_{\text {poloidal part }}+\underbrace{\left.\overrightarrow{\operatorname{rot}}\left(\psi \frac{\vec{g}}{g}\right)\right)}_{\text {toroidal part }}
$$




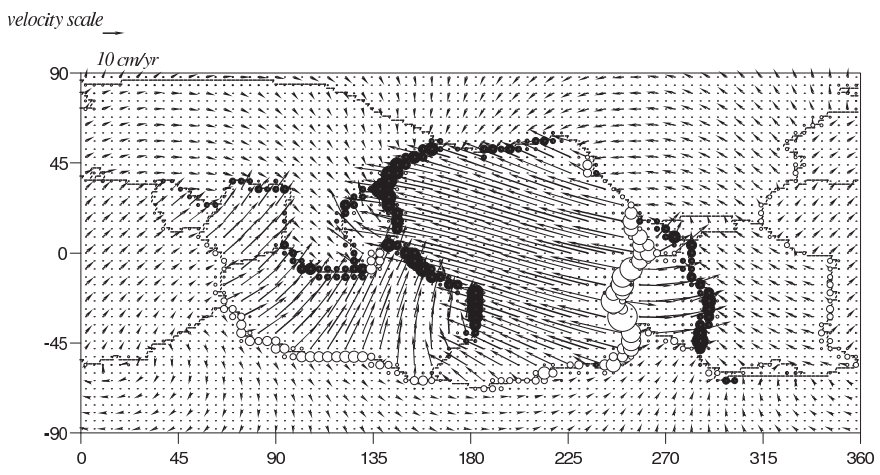

Figure 1: Present-day plate motions (NNR NUVEL1 model [1]). The black and white discs correspond to negative and positive vertical velocities, which refer to subduction zones and oceanic ridges, respectively.

The toroidal field describes lateral motions around a vertical axis and the poloidal field depicts vertical motions around a lateral axis. $\phi$ and $\psi$ can be expressed by spherical harmonics:

$$
\left\{\begin{array}{l}
\psi(r, \lambda, \varphi)=\sum_{l, m} T_{l, m}(r) Y_{l, m} \\
\phi(r, \lambda, \varphi)=\sum_{l, m} S_{l, m}(r) Y_{l, m}
\end{array}\right.
$$

A simple calculation shows that the poloidal $\left(S_{l, m}(r)\right)$ and toroidal $\left(T_{l, m}(r)\right)$ scalar fields are directly related to the horizontal divergence and the radial vorticity, respectively [2]:

$$
\overrightarrow{\nabla_{H}} \cdot \vec{v}=-\sum_{l, m} \frac{l(l+1) \dot{S}_{l, m}(r) Y_{l, m}}{\rho r^{2}}, \quad(\overrightarrow{r o t} \vec{v}) \cdot \overrightarrow{e_{r}}=\sum_{l, m} \frac{l(l+1) T_{l, m}(r) Y_{l, m}}{\rho r^{2}}
$$

\section{Degree variance of horizontal divergence and radial vorticity}

The spectra of the present-day surface horizontal divergence (triangles) and radial vorticity (circles) are plotted as a function of degree 1 in fig. 2 . The coefficients of these spectra are deduced by a multiplication by $l(l+1)$ of the corresponding poloidal and toroidal variances in eqn (3). We present the spectra for the highest wavelengths till degree $l=32$. As the reference frame is in the NNR (No Net Rotation reference frame), the radial vorticity (or toroidal field of degree one) is null. This field corresponds to a pure lithospheric rotation and does not exist in the NNR reference frame. The spectrum of the divergence is mainly located above the spectrum of the radial vorticity.

The divergence spectrum shows two main peaks for $l=4,5$ and a smaller peak for $l=2$. The vorticity spectrum provides two main peaks for $l=2,5$. The horizontal half-wavelength, $\lambda / 2$, that corresponds to a spherical harmonic degree 1 


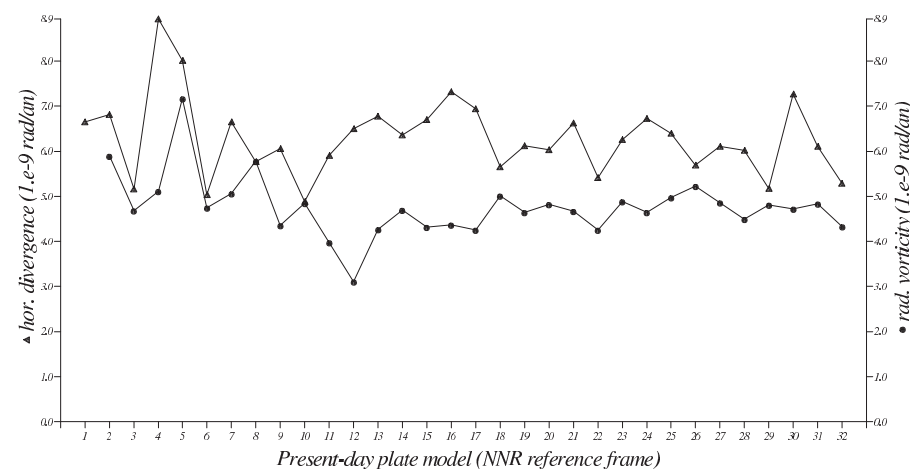

Figure 2: Variances of the horizontal divergence (triangles) and radial vorticity (circles) of present-day plate motions (NNR NUVEL1 [1]).

is given by [3]:

$$
\lambda / 2=\frac{R \pi}{(l+1 / 2)}
$$

with $R$, the radius of the Earth.

For $l=4,5$, the half-wavelengths are $4500 \mathrm{~km}$ and $3600 \mathrm{~km}$, respectively. The dominant large scale flow that drives the plates is $4000 \mathrm{~km} \mathrm{[4].} \mathrm{It} \mathrm{was} \mathrm{therefore}$ suggested by Forte and Peltier [2] that the dominant half-wavelengths of the horizontal divergence correspond to the dominant large scale flow of the mantle if the convective cells were of ratio one (the mantle depth is about $3900 \mathrm{~km}$ ). The second hypothesis that explained the high peaks observed in the horizontal divergence for degree $l=4,5$ was the Pacific plate, i.e., the Plate with the highest kinetic energy) [5].

Figures 3 and 4 present the time-dependent evolution of the horizontal divergence and radial vorticity during the Cenozoic periods for the two published plate reconstructions (Gordon and Jurdy, 1986 [6]; Torsvik et al., 2010 [7]).

The two previous hypotheses for the highest peaks are now put under large doubt as during the Cenozoic period (the past $64 \mathrm{Myr}$ ), the main peaks were not always corresponding to the degree $l=4,5$ (cf. fig. 3 and fig. 4). Moreover, the Pacific plate was the plate with the highest kinetic energy during the past $30 \mathrm{Myr}$ (because of its large surface and high velocity), but the highest peaks were not always corresponding to the degree $l=4,5$ even if the Pacific plate shape did not change fundamentally.

The spectra presented in the two plate reconstructions for similar periods are quiet different. The main differences come from the hypotheses used to build these plate motion reconstructions. The Gordon and Jurdy model [6] was based on three main postulates which have recently been placed into considerable doubt: the fixity of the Pacific hotspots, a plate motion reorganization of the Pacific plate used to explain the Hawaiian Emperor Bend and a simplified plate geometry (introduction of the Chatham Rise plate to divide the Pacific plate into two parts). The up-to-date 

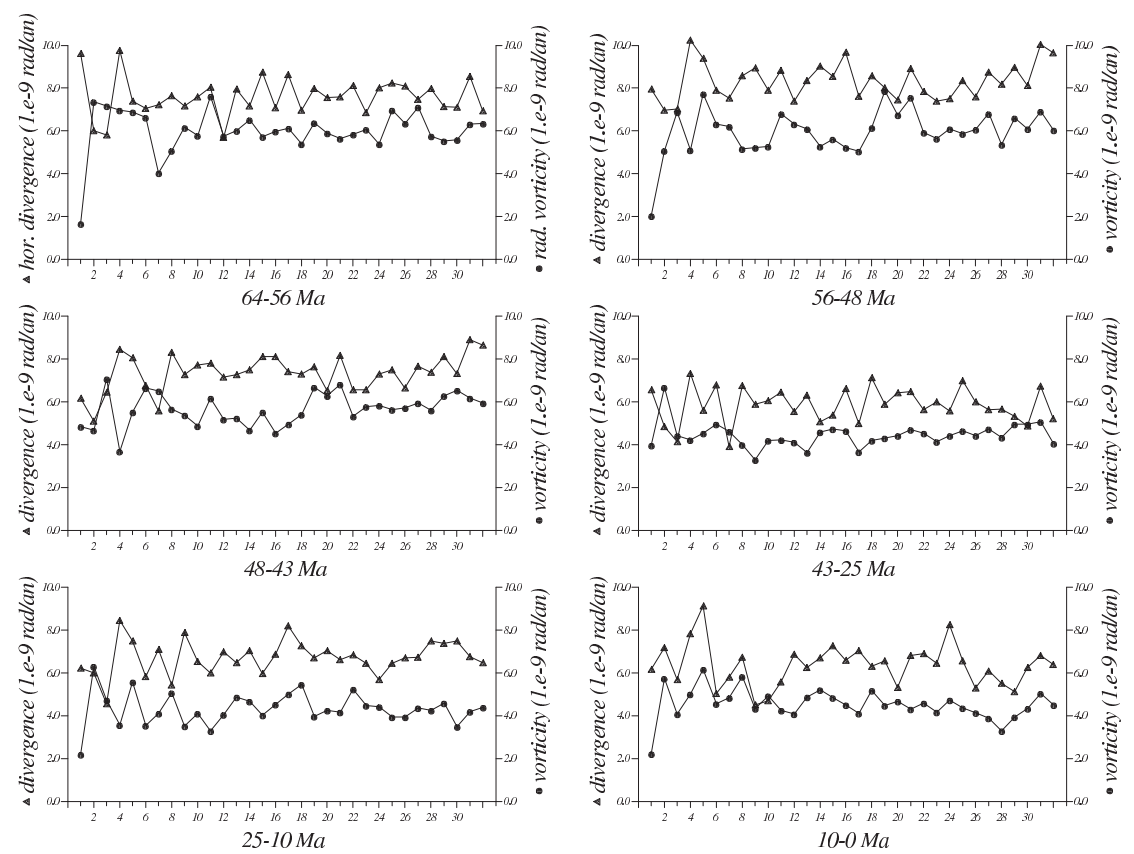

Figure 3: Variances of the horizontal divergence and radial vorticity of Cenozoic plate motions in the Gordon and Jurdy model [6].

Torsvik et al. model [7] does not use those hypotheses. Moreover, the number of plates and their geometries are different. Those plate motion uncertainties explain why the spectra are different for similar periods.

\section{New explanation for the main peaks obversed in the horizontal divergence}

The total kinetic energy is the sum of the poloidal and toroidal kinetic energies. At present-day, the plate which has the largest poloidal kinetic energy is the Pacific plate $(37 \%)$, followed by the Australian plate $(24 \%)$ and the European plate $(9 \%)$. Figure 5 presents the spectrum of the horizontal divergence for the Pacific, Australian and European plates. Even if it is not possible to add linearly the contributions of those three plates to the total horizontal divergence, it is obvious that the plates which have the largest poloidal kinetic energies will have the most contribution to the horizontal divergence. We note that at present-day, the highest peak provided by the Pacific plate is the degree 2 . The degree $l=4,5$ are mainly due to the Pacific and Australian plate. According to our new explanation, each high peak is not associated with a particular plate. The highest peaks correspond to the sum of the peaks of the plates that have the highest polo idal kinetic energy. 

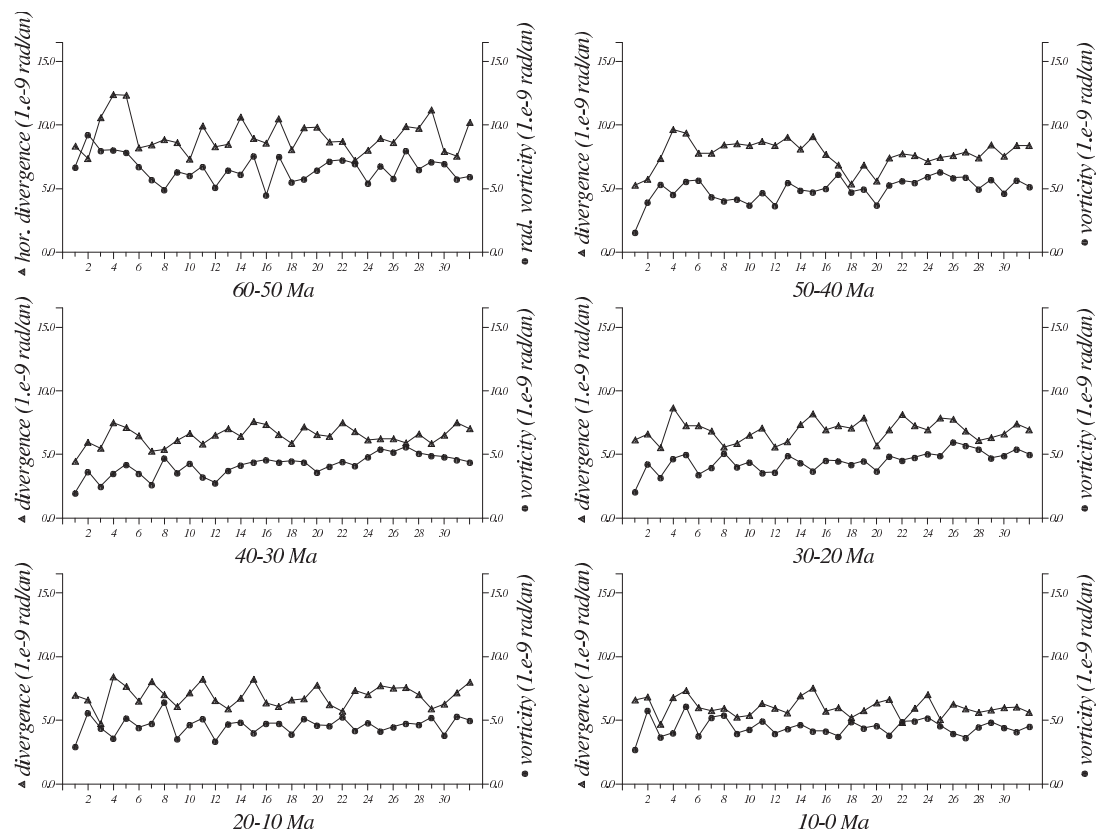

Figure 4: Variances of the horizontal divergence and radial vorticity of Cenozoic plate motions in the Torsvik et al. model [7].

\section{Mantle convection}

We model buoyancy flow by solving the equations for mass, momentum and energy conservation in an infinite Prandtl number, Boussinesq fluid.

The non-dimensionalized mass, momentum, and energy equations governing the flow take the form:

$$
\begin{gathered}
\boldsymbol{\nabla} \cdot \mathbf{v}=0 \\
-\boldsymbol{\nabla} P_{d}+R a T \hat{\mathbf{r}}+\nabla \cdot \tau=0 \\
\partial T / \partial t+\mathbf{v} \cdot \boldsymbol{\nabla} T=\nabla^{2} T+Q
\end{gathered}
$$

where $\mathbf{v}$ is the velocity vector, $P_{d}$ is the dynamical pressure, $T$ is the temperature field, $\tau$ is the deviatoric stress tensor, $R a$ is the Rayleigh number, and $Q$ is the dimensionless internal heating.

We non-dimensionalise the conservation equations according to the following relations:

$$
r=r / d, t=t \kappa / d^{2}, v=v d / \kappa \text { and } T=\left(T-T_{t o p}\right) / \triangle T
$$

with $\kappa=610^{-7} \mathrm{~m}^{2} \mathrm{~s}^{-1}, g=10 \mathrm{~ms}^{-2}, \triangle T=2000 \mathrm{~K}, \mathrm{~d}=2891 \times 10^{3} \mathrm{~m}$ and $T_{\text {top }}=0$ (the parameters are from Quéré and Forte, 2006 [8]). 

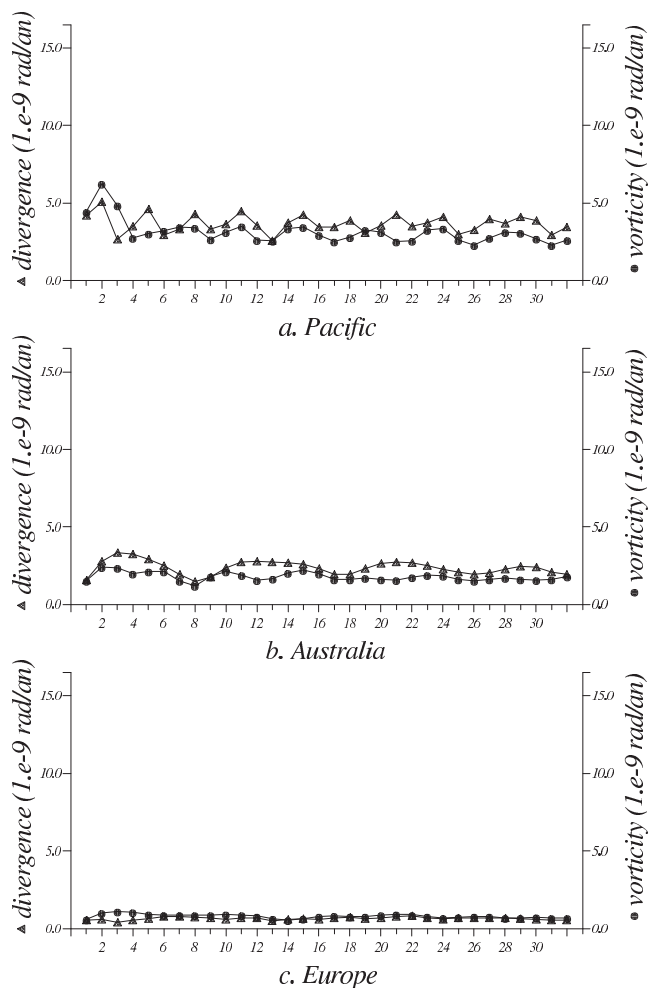

Figure 5: Degree spectrum of the horizontal divergence for the Pacific (a), Australian (b) and European (c) plates.

$R a$ is the Rayleigh number and is given by [9]:

$$
\frac{\alpha g d^{3} \rho \triangle T}{\kappa \eta}
$$

\section{Convection with time-dependent plate histories}

It is important to asses the robustness of convective results, particularly with regard to the dynamics and configuration of hot mantle upwellings. To this end, we carried out two different convection simulations incorporating the past $120 \mathrm{Myr}$ history of tectonic plate evolution, a time period for which reliable reconstructions of the plate motions are possible.

We employ a 3-D, spherical-shell, numerical model of time-dependent thermal convection in a mantle which is treated as an incompressible viscous fluid. One distinct and fundamentally important feature of our convection modelling is the ability to dynamically couple the surface plate motions to the buoyancy driven 
forces in the mantle. With the exception of Monnereau and Quéré (2001) [10] and Quéré and Forte (2006) [8], previous investigations of time-dependent thermal convection in 3-D spherical geometry do not implement a dynamically consistent coupling of plates and mantle flow. The plates are coupled to the mantle using a torque balancing procedure which depends on the flow-induced viscous stresses generated by the internal mantle flow and the flow associated with the surface plates (e.g., Monnereau and Quéré (2001) [10]). This method can be used either with imposed plate motions or with freely moving plates in response to the mantle flow below.

We first derive a reference convection mode using the plate motions 120 Ma till a steady state is obtained. This reference model used the parameters from Quéré and Forte (2006) [8] which ensured the retrieval (to within $\pm 10 \%$ ) of the main global observables at the Earth's surface (mean plate velocity, global heat flux and potential temperature under oceanic ridge). We then impose the plate motion reconstruction that span the past $120 \mathrm{Myr}$ (Mesozoic and Cenozoic periods). We compare the results obtained with the two main plate motion reconstructions at present-day.

The Gordon and Jurdy plate motions [6] provides five focused upwellings $120 \mathrm{Ma}$ (fig. 6). As we employ a NNR reference frame and as we span only $120 \mathrm{Myr}$ of plate motion reconstruction, the location of the hotspots will not have enough time to vary during this period. At present-day, the location of those five hotspots could correspond to the location of the Caroline, Easter, Azores, Cap Verde and Kerguelen hotspots.

At present-day, the Torsvik et al. model [7] provides only two hotspots that barely correspond to known hotspots (fig. 7). However, we can not affirm using only the location of the present-day hotspots that the Gordon and Jurdy plate reconstruction is better than the Torsvik et al. model because we now know that the former one was based on false assumptions. We found that subducted material will take about $100 \mathrm{Myr}$ to descend to the bottom of the mantle and, about 50 Myr later, hot plume material will begin to erupt from the lower thermal boundary layer at the Core-Mantle-Boundary (C.M.B.). $150 \mathrm{Myr}$ seems to be the minimum time period of Earth's history to permit hotspots motions at the C.M.B. This study advocates for more precise plate reconstructions that would span the past $400 \mathrm{Myr}$ of Earth's history to ensure the observation of meaningful hotspots motions at the C.M.B.

\section{Constructal theory, plate tectonics and chaos}

According to several observations, plate tectonics seems to be constructal (Quéré, 2010, [11]). Thanks to the constructal theory created by Bejan [12], dynamics of various natural processes is determined by the self-optimization of material and energy flows in the system. This idea, which can be put into a context of variational and extremal principles of physics (such as Least Action Principle, Energy Minimization Principle, or Maximum Entropy Principle), allows for a broad exploratory latitude in actual modeling of the processes. 

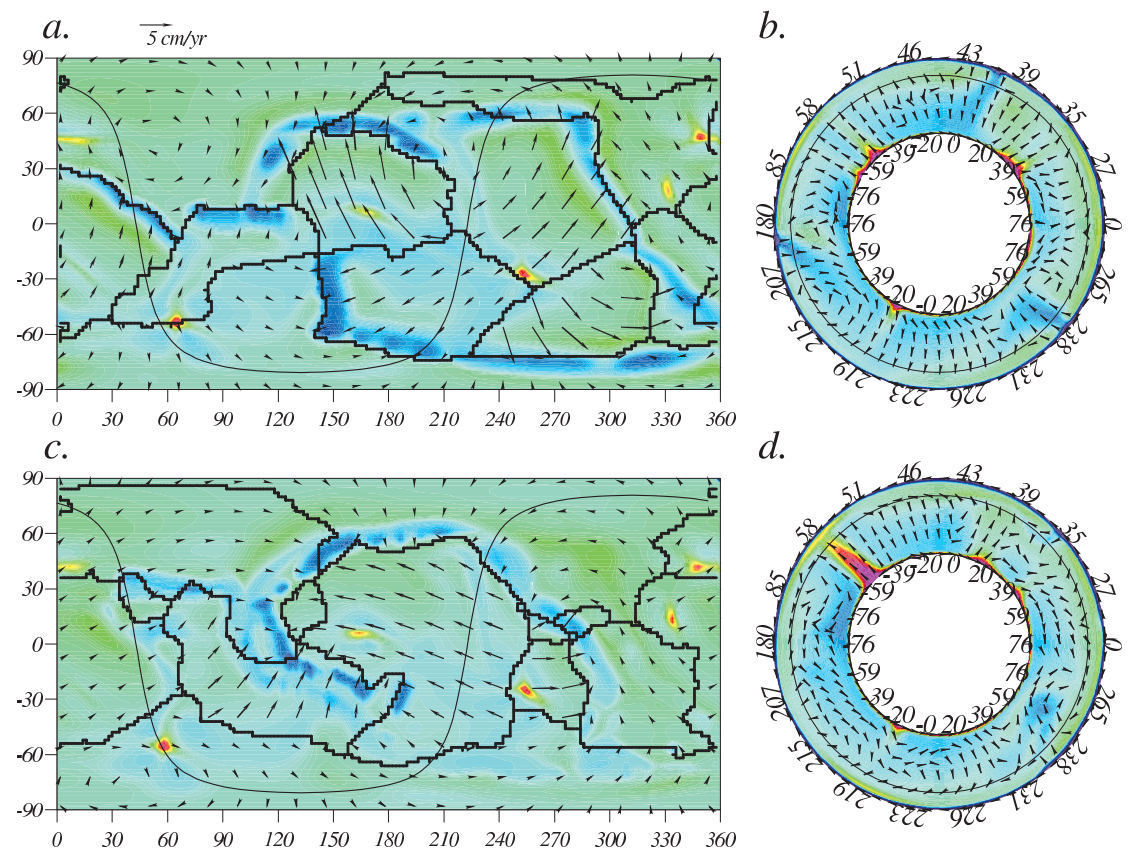

$\begin{array}{lllllllllll}0.0 & 0.1 & 0.2 & 0.3 & 0.4 & 0.5 & 0.6 & 0.7 & 0.8 & 0.9 & 1.0\end{array}$

Figure 6: Simulation of the past 120 Myr using the Gordon and Jurdy model. (a) The lateral temperature variations at mid-mantle $120 \mathrm{Ma}$. The surface velocity field (black arrows) and surface plate boundaries (solid black lines) are superimposed. (b) Corresponding whole- mantle cross-section $120 \mathrm{Ma}$, along dashed line in (a), showing mantle temperature with the velocity field (black arrows) super- imposed. (c) and (d) correspond to the end of the simulation at present-day.

As we saw previously, mantle convection is characterized by the Rayleigh number $R a$. The $R a$ number for mantle convection is about $10^{6}-10^{7}$, i.e., more than one or ten thousand times the critical $R a$ for which thermal convection begins. Within this range of $R a$ number, even if compressibility and internal heating are taken into account, mantle convection is still governed by chaos, i.e., the system becomes highly unpredictable. The main features of a chaotic general behavior are the (1) sensitivity to initial conditions also known as the Butterfly effect and (2) the rapid mixing of the model solutions or phase trajectories, i.e., the ergodicity principle.

In case of the Earth, the unpredictability results from the sensitivity to initial conditions (accretion of the proto-Earth or the random initial conditions of a computational simulation). Once chaos has started, the chaotic behavior 

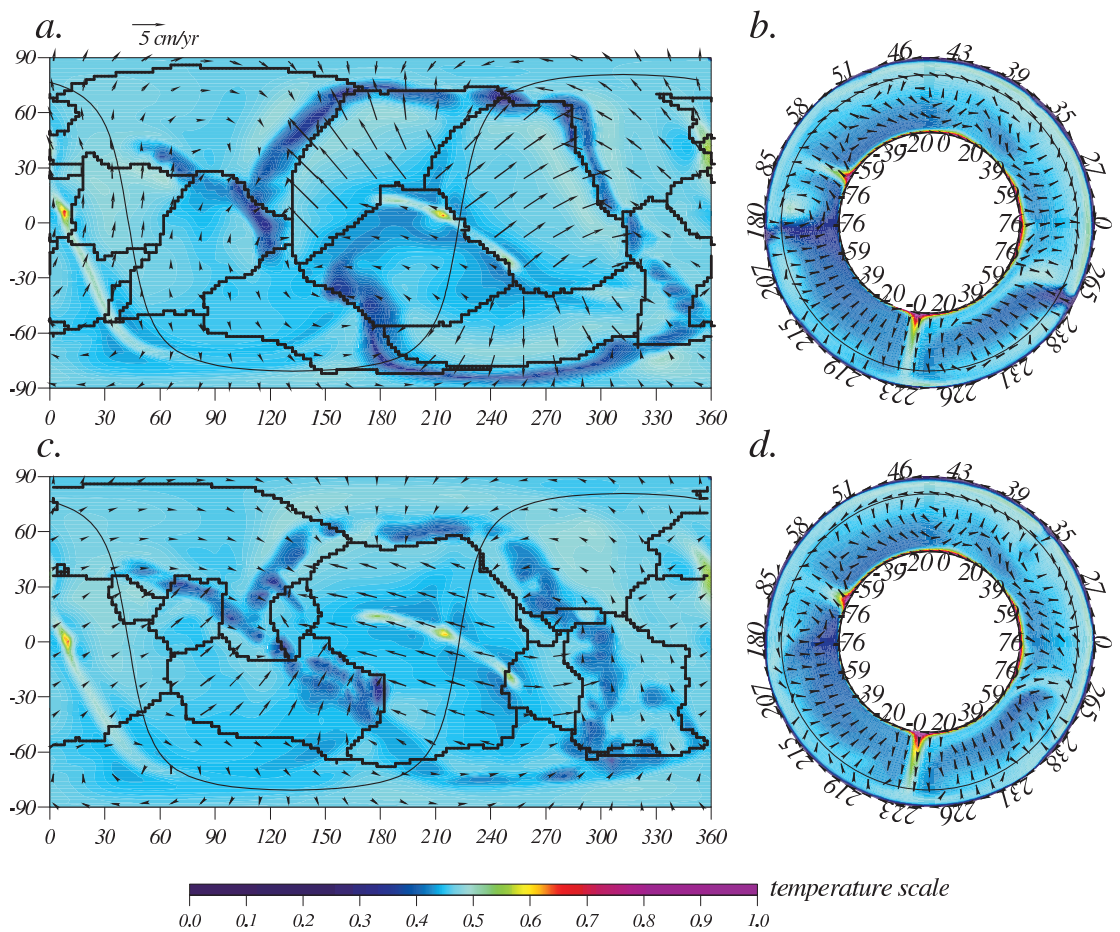

Figure 7: Analogous to Figure 6 but for a simulation using the Torsvik et al. model [7].

persists for $R a$ analogous to the terrestrial $R a$. A calculation of the exact image of an Earths'interior is therefore impossible. However, chaotic behaviors have interesting statistical properties: the high scale structures are conserved even if local and instantaneous effects are unpredictable, such as temperature or velocity. In brief, by knowing the instantaneous value of the velocity at a particular point, it is impossible to predict the future value of the velocity at the same point.

Typical signature of this kind of chaos is the presence of a 'strange attractor' which is a particular arrangement of the phase trajectory showing fractal structure [13-15] (simpler attractors with less dimensions would be simply a point, a periodic cycle or a torus). Plate motion reconstructions depict plate motions and geometry which are evolving slowly in time in coherence with the underlying chaotic mantle flow. We therefore argue that plate motions are chaotic as they are directly linked to mantle convection.

\section{Conclusions}

The present-day plate motions are accurate due to the recent data used to build the NNR NUVEl1 model. In the past, uncertainties prevent us from knowing how 
the plates evolved with exactitude. However, the past motions enable us to confirm that the main peaks observed for the horizontal divergence at present-day are not explained by convective cells of ratio one, neither uniquely by the Pacific plate, but merely by the contribution of the main plates that add to the kinetic energy. Due to the uncertainties in building past plate motions, when we use the two published plate reconstructions on top of a 3D convective code where plates are dynamically linked to the mantle, we observe different locations for the hotspots at present-day. This study shows the necessity of having more precise plate reconstructions that could span the past $400 \mathrm{Myr}$.

Mantle flow is governed by chaos. As plate tectonics correspond to the surface boundary layer of mantle flow, plate motion reconstructions are likely to be subject to a chaotic and constructal behavior.

\section{References}

[1] Argus, D. \& Gordon, R., No net rotation model of current plate velocities incorporating plate motion model NUVEL-1, Geophys. Res. Lett., 18, pp. 2039-2042, 1991.

[2] Forte, A.M. \& Peltier, W.R., Plate tectonics and aspherical Earth structure: The importance of poloidal-toroidal coupling, J. Geophys. Res., 92, pp. 36453679, 1987.

[3] Lapwood, E.R. \& Usami, T., Free Oscillations of the Earth, New York, Cambridge University Press, 1981.

[4] Peltier, W.R, Surface Plates and Thermal Plumes: Separate Scales of The Mantle Convective Circulation.. Eds. R.J. O'Connell and W. Fyfe, Geodyn. Ser., 6. A.G.U. Publications, 1981.

[5] O’Connell, R.J., Gable, C.W. \& Hager, B.H., Toroidal-poloidal partitioning of lithospheric plate motions, in Glacial Isostasy, Sea Level and Mantle Rheology, Ed. by R. Sabadini and K. Lambeck, Klumer Academi Publishers, Dordrecht, 1991.

[6] Gordon, R.G. \& Jurdy, D.M., Cenozoic global plate motions, J. Geophys. Res., 91, 12389 (1986).

[7] Torsvik, T.H., Steinberger, B., Gurnis, M. \& Gaina, C., Plate tectonics and net lithosphere rotation over the past $150 \mathrm{Myr}$, Earth planet. Sci. Lett., 291, pp. 106-112, 2010.

[8] Quéré, S. \& Forte, A.M., Influence of past and present-day plate motions on spherical models of mantle convection: Implications for mantle plumes and hotspots, Geophys. J. Int., 165, pp. 1041-1057, 2006.

[9] Chandrasekhar, S., Hydrodynamic and Hydromagnetic Instability, Clarendon Press, 1961.

[10] Monnereau, M. \& Quéré S., Spherical shell models of mantle convection with tectonic plates, Earth planet. Sci. Lett., 184, pp. 575-587, 2001.

[11] Quéré, S., Constructal theory of plate tectonics, Int. J. of Design \& Nature and Ecodynamics, Vol. 5, No. 3 (2010), 242-253. 
[12] Bejan, A., Shape and Structure, from Engineering to Nature, Cambridge University Press, Cambridge, UK, 2000, 324 p. ISBN 0521793882.

[13] Grassberger, P. \& Procaccia, I., Characterization of Strange Attractors, Physical Review Letters, 50, pp. 346-349, 1983.

[14] Grassberger, P. \& Procaccia, I., Measuring the Strangeness of Strange Attractors, Physica 9D, pp. 189-208, 1983.

[15] Berezin, A.A., Isotopic Relative of Strange Attractors, Physics Letters, A161, 295-300 (1991). 